'Departamento de Psicología y Centro Vida Saludable, Universidad de Concepción. Concepción, Chile.

2Instituto de Anatomía, Histología y Patología, Facultad de Medicina, Universidad Austral de Chile. Valdivia, Chile.

${ }^{3}$ CIEDE-UCSC, Facultad de Medicina, Universidad Católica de la Santísima Concepción. Concepción,

Chile.

${ }^{4}$ Instituto de Farmacia, Facultad de Ciencias, Universidad Austral de Chile. Valdivia, Chile. ${ }^{5}$ Institute of Health and Wellbeing, University of Glasgow. Glasgow, United Kingdom. ${ }^{6}$ Departamento de Ciencias Básicas. Universidad Católica de la Santísima Concepción. Concepción, Chile.

${ }^{7}$ Departamento de Nutrición y Dietética. Facultad de Farmacia, Universidad de Concepción. Concepción, Chile.

${ }^{8}$ Departamento de Bioquímica Clínica e Inmunología, Facultad de Farmacia y Centro de Vida Saludable de la Universidad de Concepción. Concepción, Chile.

${ }^{9}$ Grupo de Investigación Calidad de Vida, Departamento Ciencias de la Educación, Universidad del Biobío. Chillán, Chile.

${ }^{10}$ Escuela de Kinesiología, Facultad de Salud, Universidad Santo Tomás, Sede Valdivia. Valdivia, Chile.

'Departamento de Ciencias del Deporte y Acondicionamiento Físico, Facultad de Educación, Universidad Católica de la Santísima Concepción. Concepción, Chile.

${ }^{12} \mathrm{BHF}$ Glasgow Cardiovascular Research Centre, Institute of Cardiovascular and Medical Science, University of Glasgow. Glasgow, United Kingdom.

${ }^{13}$ Centro de Investigación en Fisiología del Ejercicio-CIFE, Universidad Mayor. Santiago, Chile. aPsicóloga. bProfesora de Biología y Química. 'Nutricionista. dBioquímico. eProfesor de Educación Física. ${ }^{\mathrm{f}} \mathrm{PhD}$. ${ }^{9}$ Msc.

Trabajo no recibió financiamiento. Los autores declaran no tener conflictos de interés.

Recibido el 25 de septiembre de 2018, aceptado el 7 de noviembre de 2019

Correspondencia a:

Dr. Carlos Celis-Morales

Glasgow Cardiovascular Research Centre Institute of Cardiovascular and Medical Science University of Glasgow. G12 8TA. Glasgow, United Kingdom. Carlos.Celis@glasgow.ac.uk

\section{¿Cuál es la asociación entre el tiempo destinado a dormir y el desarrollo de deterioro cognitivo en adultos mayores chilenos?}

\author{
GABRIELA NAZAR ${ }^{1, \mathrm{a}, \mathrm{f}}$, ANA MARÍA LEIVA ${ }^{2, \mathrm{~b}, \mathrm{~g}}$, \\ CLAUDIA TRONCOSO ${ }^{3, \mathrm{c}, \mathrm{g}}$, ADELA MARTÍNEZ $^{4, \mathrm{~d}, \mathrm{~g}}$, \\ FANNY PETERMANN-ROCHA ${ }^{5, \mathrm{c}, \mathrm{g}}$, MARCELO VILLAGRÁN $^{6, \mathrm{~d}, \mathrm{f}}$, \\ LORENA MARDONES ${ }^{6, \mathrm{~d}, \mathrm{f}}$, MIQUEL MARTORELL ${ }^{7, \mathrm{~d}, \mathrm{f}}$, \\ ANA MARÍA LABRAÑA ${ }^{7, \mathrm{c}, \mathrm{g}}$, NATALIA ULLOA ${ }^{8, \mathrm{~d}, \mathrm{f}}$, \\ XIMENA DÍAZ-MARTÍNEZ ${ }^{9, e, g}$, FELIPE POBLETE- \\ VALDERRAMA $^{10, e, g}$, ALEX GARRIDO-MÉNDEZ $^{11, e, f}$, \\ CARLOS CELIS-MORALES ${ }^{12,13, e, f}$, en representación \\ del Grupo de investigación ELHOC.
}

\section{Association between sleep duration and cognitive impairment in older people}

Background: Sleep duration may be a risk factor for cognitive impairment. Aim: To investigate the association between sleep duration and cognitive function in Chilean older adults. Material and Methods: We analyzed information from 1,384 participants aged $>60$ years participating in the National Health Survey 2009-2010 who were assessed with the Mini Mental State Examination (MMSE) and self-reported their average daily sleep hours. Logistic regression analysis was performed to investigate the association between MMSE and sleep duration. Results: Compared to those participants who reported sleeping 7 hours per day, those that reported sleeping $<5$ hours had a higher odd for cognitive impairment (Odds ratio (OR): 3.66 [95\% confidence intervals (CI: 1.69; 7.95], $p<0.01$ ). Similarly, those who reported sleeping $>8$ hours per day also showed a higher odd for cognitive impairment (OR: 2.56 [95\% CI: 1.32; 4.95], $p<0.01$ ). This association was even stronger for people who reported more than 10 hours of sleep per day (OR: 4.46 [95\% CI: 1.32; 4.95], $p<0.01$ ). Conclusions: Long and short sleep duration is associated with cognitive impairment in older adults in Chile independent of major confounding factors.

(Rev Med Chile 2019; 147: 1398-1406)

Key words: Aged; Cognitive Dysfunction; Sleep. 
C onsiderando el progresivo envejecimiento de la población, se espera que el número de personas con alteraciones cognitivas aumente progresivamente en los próximos años ${ }^{1}$. A nivel mundial, 9\% de la población de 60 años presenta algún grado de deterioro cognitivo, el cual se incrementa a $20 \%$ en personas mayores a 80 años $^{2}$. Entre las patologías asociadas con el deterioro cognitivo está la demencia, y se estima que 1 de cada 4 personas con deterioro cognitivo podría desarrollar algún tipo de demencia en su vida adulta $^{3}$. Si bien en el 2010 se estimó 35,6 millones de personas con demencia, se espera que esta cifra aumente a 81,1 millones para el $2040^{1}$. Lo anterior significará un incremento en el costo económico directo e indirecto asociado a esta enfermedad. Al respecto, el año 2000 el costo de la demencia alcanzó $\$ 280$ billones de dólares, cifra que aumentó a $\$ 948$ billones de dólares en el $2016^{4}$ y se espera siga incrementando.

El escenario en Chile no es alentador, la Encuesta Nacional de Salud 2009-2010 (ENS 20092010 ) indica que $10,4 \%$ de la población mayor de 60 años presenta deterioro cognitivo, cifra que aumenta a $20,9 \%$ en personas de 80 años y más ${ }^{5}$. En la actualidad en Chile cerca de 200.000 personas viven con demencia. Si cada una integra una familia de cuatro integrantes, las personas relacionadas con esta condición aumentan a casi un millón lo cual refleja el costo social y familiar de esta enfermedad ${ }^{6}$. En este contexto, se hace necesario incrementar los esfuerzos orientados a la prevención del deterioro cognitivo y aminorar los diversos costos asociados.

Estudios han abordado los antecedentes de alteraciones cognitivas, incluidas deterioro cognitivo y demencias ${ }^{1,3,7,8}$. Entre los principales predictores se encuentran factores no modificables como la genética (gen EPOE4) y edad, pero también una serie de factores modificables como baja escolaridad, aislamiento social, hipoacusia, obesidad, inactividad física, diabetes mellitus tipo 2 (DMT2), hipertensión arterial (HTA), depresión $\mathrm{y}$ otras enfermedades mentales no tratadas ${ }^{7-9}$. Si bien cada uno de estos factores contribuye al desarrollo de demencia, su rol varía a través del ciclo vital'. Un reciente reporte de la revista "Lancet", plantea que el nivel educacional es un factor de riesgo relevante en la etapa infantil, mientras que la obesidad, HTA e hipoacusia son factores de riesgo más importantes en la etapa adulta (30 a 50 años), y el tabaquismo, DMT2, depresión, aislamiento e inactividad física relevantes durante la tercera edad ${ }^{9}$. Sin embargo, todos estos factores solo explican 35\% del riesgo total de desarrollar demencia, por lo cual aún existen otros riesgos emergentes que podrían contribuir al desarrollo de esta patología y para los cuales existe una limitada cantidad de evidencia9.

Uno de los factores asociados a la presencia de alteraciones cognitivas que ha recibido atención durante los últimos años es el sueño ${ }^{10}$, particularmente el número de horas dormidas diariamen$\mathrm{te}^{11}$. Estudios previos han identificado que horas extendidas y disminuidas de sueño se relacionan con un funcionamiento cognitivo disminuido en personas mayores de 30 años $^{12}$. Estudios en personas $\geq 65$ años han identificado que horas de sueño $\geq 7 y \geq 8$ se asocian con puntuaciones disminuidas en funcionamiento cognitivo ${ }^{13}$; otros indican que $\leq 5 \mathrm{~h} \mathrm{o} \geq 9 \mathrm{~h}$ de sueño diarias se asocian con un peor funcionamiento cognitivo global ${ }^{11,14}$. A pesar de esta evidencia, en Chile la asociación entre horas destinadas a dormir y deterioro cognitivo no ha sido estudiada. Considerando que la población chilena duerme en promedio $6,8 \mathrm{~h}$ (inferior a la recomendación de 7-9 h/día) junto con el aumento progresivo de la población adulta mayor en Chile, este estudio se propuso investigar la asociación entre horas destinadas a dormir y deterioro cognitivo en adultos mayores chilenos.

\section{Material y Método}

\section{Diseño del estudio}

La muestra seleccionada comprende a todos los participantes mayores de 60 años de la ENS 2009-2010 que poseían información sobre funcionamiento cognitivo ${ }^{5}$. La ENS 2009-2010 correspondió a un estudio de prevalencia realizado en hogares en una muestra nacional, probabilística, estratificada y multietápica de 5.412 personas mayores de 15 años con representatividad nacional, regional, y área urbana/rural. En este estudio fueron incluidos 1.384 participantes mayores de 60 años (61\% mujeres). Para este estudio se clasificó como adulto mayor a todas las personas $\geq 60$ años de edad en base a las recomendaciones de inicio del envejecimiento de la $\mathrm{OMS}^{15}$. El protocolo de estudio fue aprobado por el Comité de Ética de la Escuela de Medicina de la Pontificia Universidad 
Católica de Chile. Todos los participantes firmaron un consentimiento informado ${ }^{5}$.

\section{Evaluación de deterioro cognitivo}

El funcionamiento cognitivo fue evaluado a través del Mini Mental State Examination $(\mathrm{MMSE})^{16}$ instrumento de screening de deterioro cognitivo. Para este estudio, se utilizó la versión abreviada de 6 preguntas, con un puntaje máximo de 19 puntos y punto de corte mayor o igual a 13 puntos. Esta versión ha sido previamente validada en población chilena ${ }^{17,18}$.

Los datos de tiempo destinado a dormir se obtuvieron a través de las preguntas abiertas: a) En promedio ¿cuántas horas duerme los días de semana y b) En promedio ¿cuántas horas duerme los fines de semana? El promedio en horas de las dos medidas reportadas (semana y fin de semana) fue utilizado como variable de exposición en este estudio ${ }^{5}$.

Las variables sociodemográficas (edad, sexo, nivel educacional, zona geográfica) y datos de control asociados con estilo de vida, como tabaquismo, consumo de alcohol, frutas y verduras y auto-reporte de salud y bienestar, se obtuvieron mediante la aplicación de cuestionarios validados en la ENS 2009-2010.

El estado nutricional fue determinado a partir del índice de masa corporal (IMC: $\mathrm{kg} / \mathrm{m}^{2}$ ), utilizando los puntos de corte de valoración específica para el adulto mayor: bajo peso: $<22,9 \mathrm{~kg} / \mathrm{m}^{2}$; normo peso: $23,0-27,9 \mathrm{~kg} / \mathrm{m}^{2}$; sobrepeso: 28,0 $31,9 \mathrm{~kg} / \mathrm{m}^{2}$ y obesidad: $\geq 32,0 \mathrm{~kg} / \mathrm{m}^{226}$, mientras que la obesidad central fue definida como un perímetro de cintura $\geq 88 \mathrm{~cm}$ para mujeres $\mathrm{y} \geq 102 \mathrm{~cm}$ para hombres, según los puntos de corte sugeridos por la ENS 2009-20105. Los niveles de actividad física (AF), el tiempo destinado a las actividades de transporte activo y las actividades de intensidad moderada o vigorosa fueron determinados con el cuestionario "Global Physical Activity Questionnaire"19. La AF total es presentada como la suma del tiempo reportado en actividades de transporte, de intensidad moderada y vigorosa en el trabajo y en el tiempo libre. Se consideró como punto de corte para inactividad física un gasto energético < $600 \mathrm{METs} / \mathrm{min} / \mathrm{semana}$. Los niveles de sedentarismo fueron determinados a partir del auto-reporte de tiempo destinado a actividades que involucren estar sentado o reclinado durante el tiempo libre o de trabajo.

\section{Análisis estadístico}

Los datos de caracterización de la población fueron analizados como promedio y desviación estándar para variables continuas, y como porcentaje para variables categóricas, con sus respectivos $95 \%$ de intervalo de confianza (95\%IC). Diferencias significativas entre personas con y sin sospecha de deterioro cognitivo fueron determinadas con t-test para variables contínuas y con Chi-cuadrado para variables categóricas. Para categorías de horas de sueño se realizaron análisis de tendencia para determinar si el promedio o prevalencia de las variables estudiadas incrementaban o disminuían según horas de sueño.

Para investigar la asociación entre MMSE alterado (sospecha de deterioro cognitivo) y horas de sueño, se realizaron análisis de regresión logística ajustadas por edad, sexo, nivel educacional, tabaquismo, IMC, DMT2, HTA consumo de alcohol y consumo de frutas y verduras.

Para los análisis se utilizó el módulo de análisis de muestras complejas del programa STATA SE v14 y todos los resultados fueron estimados utilizando muestras expandidas según la ENS 2009-2010. El nivel de significancia fue definido como $\mathrm{p}<0,05$.

\section{Resultados}

La caracterización sociodemográfica de la muestra según MMSE se presenta en la Tabla 1. Del total de las personas evaluadas, 12,2\% [95\% IC: 10,$5 ; 14,0]$ presentó MMSE alterado. En general, los adultos mayores con un MMSE alterado presentaron una mayor edad ( 8,2 años), un menor nivel educacional y 2,3 veces mayor porcentaje de individuos con bajo peso en comparación a individuos con MMSE normal. En relación con el estilo de vida, personas con MMSE alterado realizaban una menor cantidad de AF y reportaron un mayor número de horas sedentes al día junto con un peor auto-reporte de salud y bienestar. Metabólicamente, los adultos mayores con MMSE alterado presentaban una mayor prevalencia de DMT2 e HTA en comparación a sus pares con MMSE normal.

Las características de la población según horas destinadas a dormir se presentan en la Tabla 2. Las personas que reportaron dormir $<7 \mathrm{~h} /$ día, comparados con quienes informan horas recomendadas 
Tabla 1. Características de la población según deterioro cognitivo

\begin{tabular}{|c|c|c|c|}
\hline & MMSE Normal & MMSE Alterado & Valor $\mathbf{P}$ \\
\hline \multicolumn{4}{|l|}{ Sociodemográficas } \\
\hline $\mathrm{n}$ & 1,215 & 169 & \\
\hline Mujeres (\%) & $61,2(58,5-63,9)$ & $59,2(51,6-66,3)$ & 0,606 \\
\hline Edad (años) & $69,9(7,4)$ & $78,1(8,9)$ & $<0,0001$ \\
\hline \multicolumn{4}{|l|}{ Zona geográfica (\%) } \\
\hline Rural & $16,4(14,4-18,6)$ & $23,7(17,8-30,7)$ & \multirow{2}{*}{0,019} \\
\hline Urbana & $83,6(81,2-85,6)$ & $76,3(69,3-82,1)$ & \\
\hline \multicolumn{4}{|l|}{ Nivel educacional (\%) } \\
\hline Básica & $59,7(56,9-62,5)$ & $86,8(80,7-91,1)$ & \multirow{3}{*}{$<0,0001$} \\
\hline Media & $32,3(29,7-35)$ & $12,0(7,8-18)$ & \\
\hline Técnico-universitaria & $8,0(6,6-9,7)$ & $1,2(0,2-4,7)$ & \\
\hline \multicolumn{4}{|l|}{ Antropométricas } \\
\hline Peso corporal (kg) & $70,5(14,2)$ & $65,8(14,6)$ & 0,0002 \\
\hline IMC $\left(\mathrm{kg} / \mathrm{m}^{2}\right)$ & $28,7(5,4)$ & $27,6(5,2)$ & 0,020 \\
\hline \multicolumn{4}{|l|}{ Estado nutricional (\%) } \\
\hline Bajo peso & $8,4(7-10,1)$ & $19,2(14-26)$ & \multirow{4}{*}{$<0,000$} \\
\hline Normal & $30(27,4-32,6)$ & $17,5(12,4-24)$ & \\
\hline Sobrepeso & $28,7(26,1-31,3)$ & $27,7(21,4-35)$ & \\
\hline Obesidad & $32,9(30,3-35,6)$ & $35,5(28,6-43,1)$ & \\
\hline Perímetro de cintura $(\mathrm{cm})$ & $95,57(12,2)$ & $94,7(13,5)$ & 0,4351 \\
\hline Obesidad abdominal (\%) & $52,6(49,9-5,5)$ & $57,3(49,8-64,7)$ & 0,249 \\
\hline \multicolumn{4}{|l|}{ Estilo de vida } \\
\hline Actividad física total (MET/h/semana) & $77,6(110,2)$ & $40,6(86,4)$ & $<0,0001$ \\
\hline Prevalencia inactividad física (\%) & $33,3(30,7-36,1)$ & $65,9(58,2-72,7)$ & $<0,0001$ \\
\hline Tiempo sedente (h/día) & $3,3(2,5)$ & $5,0(3,2)$ & $<0,0001$ \\
\hline Consumo de frutas y vegetales (g/día) & $228,2(142,4)$ & $210,3(137,0)$ & 0,155 \\
\hline Consumo de alcohol (g/día) & $40,4(70,0)$ & $39,7(41,8)$ & 0,960 \\
\hline \multicolumn{4}{|l|}{ Horas de sueño (\%) } \\
\hline$<7 \mathrm{~h}$ & $31,6(29,0-34,2)$ & $28,4(22,1-35,7)$ & \multirow{3}{*}{$<0,0001$} \\
\hline $7-9 h$ & $48,7(45,9-51,5)$ & $30,8(24,2-38,1)$ & \\
\hline$>9 \mathrm{~h}$ & $19,7(17,5-22)$ & $40,8(33,6-48,4)$ & \\
\hline \multicolumn{4}{|l|}{ Tabaquismo (\%) } \\
\hline Nunca & $51,6(48,8-54,4)$ & $59,5(51,9-66,7)$ & \multirow{3}{*}{0,031} \\
\hline Exfumador & $32,2(29,6-34,9)$ & $31,6(24,9-39)$ & \\
\hline Fumador & $16,2(14,3-18,4)$ & $8,9(5,4-14,3)$ & \\
\hline \multicolumn{4}{|l|}{ Salud } \\
\hline \multicolumn{4}{|l|}{ Auto-reporte salud y bienestar (\%) } \\
\hline Malo & $2,8(2,0-3,9)$ & $12,5(8,2-18,4)$ & \multirow{3}{*}{$<0,000$} \\
\hline Regular & $36,1(33,4-38,8)$ & $50,0(42,4-57,5)$ & \\
\hline Bueno & $61,1(58,2-63,7)$ & $37,5(30,4-45,1)$ & \\
\hline Síndrome metabólico (\%) & $51,5(47,4-55,5)$ & $54,9(43,9-65,3)$ & 0,566 \\
\hline DMT2 (\%) & $23,9(21,3-26,50$ & $33,1(25,9-41,1)$ & 0,015 \\
\hline Hipertensión arterial (\%) & $68,5(65,7-71,1)$ & $81,7(74,8-86,9)$ & 0,015 \\
\hline Puntaje mini mental & $17,0(1,9)$ & $8,5(3,4)$ & $<0,0001$ \\
\hline
\end{tabular}

Datos son presentados como promedio y desviación estándar para variables continuas, y como porcentaje, para variables categóricas, con sus respectivos 95\% IC. Diferencias para variables continuas en personas con y sin sospecha de deterioro cognitivo fueron determinadas con la prueba de t-test y con Chi-cuadrado para variables categóricas. 
Tabla 2. Características de la población según categorías de tiempo destinado a dormir

\begin{tabular}{|c|c|c|c|c|}
\hline & $<7 \mathrm{~h}$ & 7-9 h & $\geq 9 \mathrm{~h}$ & Valor P \\
\hline \multicolumn{5}{|l|}{ Sociodemográficas } \\
\hline $\mathrm{n}$ & 432 & 644 & 308 & \\
\hline Mujeres (\%) & $56,2(51,5-60,9)$ & $60,6(56,7-64,3)$ & $68,5(63,1-73,5)$ & 0,004 \\
\hline Edad (años) & $70,1(7,6)$ & $70,2(7,9)$ & $73,2(8,6)$ & $<0,0001$ \\
\hline \multicolumn{5}{|l|}{ Zona geográfica (\%) } \\
\hline Rural & $18,2(14,9-22,2)$ & $16,8(14,1-19,9)$ & $16,8(13,1-21,5)$ & \multirow{2}{*}{0,767} \\
\hline Urbana & $81,7(77,8-85,1)$ & $83,2(80,1-86,0)$ & $83,1(78,5-87,0)$ & \\
\hline \multicolumn{5}{|l|}{ Nivel educacional (\%) } \\
\hline Básica & $7,0(4,9-9,8)$ & $8,7(6,7-11,1)$ & $4,1(2,3-7,1)$ & \multirow{3}{*}{0,016} \\
\hline Media & $31,2(27,0-35,8)$ & $30,9(27,4-34,6)$ & $25,3(20,7-30,7)$ & \\
\hline Técnico-universitaria & $61,8(57,1-66,3)$ & $60,4(56,6-64,1)$ & $70,5(65,1-75,5)$ & \\
\hline \multicolumn{5}{|l|}{ Antropométricas } \\
\hline Peso corporal (kg) & $71,8(14,7)$ & $69,3(13,7)$ & $68,8(14,7)$ & 0,006 \\
\hline IMC $\left(\mathrm{kg} / \mathrm{m}^{2}\right)$ & $29,1(5,6)$ & $28,3(5,24)$ & $28,5(5,5)$ & 0,115 \\
\hline \multicolumn{5}{|l|}{ Estado nutricional (\%) } \\
\hline Bajo peso & $6,8(4,8-9,7)$ & $9,3(7,3-11,8)$ & $15,0(11,3-19,4)$ & \multirow{4}{*}{0,005} \\
\hline Normal & $27,3(23,3-31,7)$ & $31,5(28,0-35,2)$ & $23,6(19,1-28,7)$ & \\
\hline Sobrepeso & $31,3(27,1-35,9)$ & $26,8(23,5-30,4)$ & $28,2(23,4-33,6)$ & \\
\hline Obesidad & $34,6(30,2-39,2)$ & $32,3(28,8-36,1)$ & $33,2(28,1-38,8)$ & \\
\hline Perímetro de cintura $(\mathrm{cm})$ & $96,1(12,5)$ & $95,0(12,1)$ & $95,7(12,8)$ & 0,554 \\
\hline Obesidad abdominal (\%) & $56,0(51,3-60,6)$ & $53,6(49,7-57,4)$ & $48,7(43,154,3)$ & 0,151 \\
\hline \multicolumn{5}{|l|}{ Estilo de vida } \\
\hline Actividad física total (MET/h/semana) & $82,3(114,6)$ & $78,7(111,7)$ & $48,8(86,5)$ & $<0,0001$ \\
\hline Prevalencia inactividad física (\%) & $30(25,8-34,5)$ & $33,7(30,1-37,5)$ & $54,8(49,1-60,3)$ & $<0,0001$ \\
\hline Tiempo sedente (h/día) & $3,4(2,6)$ & $3,4(2,5)$ & $4(2,9)$ & 0,010 \\
\hline Consumo de frutas y vegetales (g/día) & $241,22(159,5)$ & $221,5(135,8)$ & $212,8(123,5)$ & 0,009 \\
\hline Consumo de alcohol (g/día) & $35,6(47,2)$ & $48,2(89,6$ & $29,7(26,4)$ & 0,873 \\
\hline \multicolumn{5}{|l|}{ Tabaquismo (\%) } \\
\hline Nunca & $48,4(43,7-53,1)$ & $53,0(49-56,8)$ & $57,9(52,1-63,4)$ & \multirow{3}{*}{0,093} \\
\hline Exfumador & $35,6(31,3-40,3)$ & $30,7(27,3-34,4)$ & $29,8(24,8-35,3)$ & \\
\hline Fumador & $16(12,8-197)$ & $16,3(13,6-19,4)$ & $12,3(9-16,6)$ & \\
\hline \multicolumn{5}{|l|}{ Salud } \\
\hline \multicolumn{5}{|l|}{ Auto-reporte salud y bienestar (\%) } \\
\hline Malo & $5,8(3,9-8,4)$ & $2,8(1,8,4)$ & $4,1(2,3-7,1)$ & \multirow{3}{*}{0,076} \\
\hline Regular & $38,9(34,443,6)$ & $36,2(32,6-40)$ & $39,7(34,3-45,5)$ & \\
\hline Bueno & $55,3(50,6-60)$ & $61(57,1-64,7)$ & $56,1(50,4-61,8)$ & \\
\hline Síndrome metabólico (\%) & $54,3(47,5-61)$ & $50(44,5-55,6)$ & $52,7(44,6-60,6)$ & 0,076 \\
\hline DMT2 (\%) & $22,9(19-27,3)$ & $22,7(19,4-26,4)$ & $32,8(27,4-38,7)$ & 0,004 \\
\hline Hipertensión arterial (\%) & $68,2(63,4-72,5)$ & $71(67,3-74,5)$ & $71(65,4-76)$ & 0,615 \\
\hline \multicolumn{5}{|l|}{ Puntaje mini mental } \\
\hline MMSE normal & $88,9(85,6-91,5)$ & $91,9(89,6-93,8)$ & $77,6(72,6-82)$ & \multirow[b]{2}{*}{$<0,0001$} \\
\hline MMSE Alterado & $11,1(8,5-14,4)$ & $8,1(6,2-10,4)$ & $22,4(18,1-27,4)$ & \\
\hline
\end{tabular}

Datos son presentados como promedio y desviación estándar para variables continuas, y como porcentaje para variables categóricas, con sus respectivos 95\% IC. Se determinaron valores de significancia para tendencia en variables continuas según categorías de sueño mediante análisis de regresión lineal. Diferencias para variables categóricas según deterioro cognitivo y categorías de sueño fue determinada mediante análisis de Chi-cuadrado. 
de sueño (7-9 h), presentaron mayor porcentaje de malnutrición por exceso, mayor prevalencia de síndrome metabólico y peor auto-reporte de salud. En contraparte, personas con horas extendidas de sueño $(>9 \mathrm{~h}$ ) fueron predominantemente mujeres, de mayor edad, mayor nivel de estudios, menor nivel de AF y mayor prevalencia de DMT2, comparadas con quienes informan horas recomendadas de sueño.

Al evaluar sospecha de deterioro cognitivo (MMSE alterado) según horas de sueño (Figura 1 A y Tabla 3), se evidenció que en comparación a personas que reportaron dormir en promedio $7 \mathrm{~h} /$ día (grupo de referencia) aquellas que reportaron dormir $\leq 5 \mathrm{~h} /$ día así como aquellas que reporta- ron dormir $\geq 8 \mathrm{~h}$ /día, presentaban una mayor probabilidad de sospecha de deterioro cognitivo. Quienes reportaron dormir $5 \mathrm{~h}$ /día y 4 h/día presentaron 3,7 y 4,9 veces mayor probabilidad, respectivamente, de tener deterioro cognitivo en comparación a aquellas personas que reportaron dormir $7 \mathrm{~h}$ /día. Por otra parte, quienes reportaron dormir $8 \mathrm{~h}$ /día presentaron una probabilidad 2,6 veces mayor de presentar sospecha de deterioro cognitivo, en comparación con el grupo de referencia, probabilidad que aumentó 2,7, 4,5, 10,3 y 15,2 veces para quienes informaron dormir 9 , 10,11 y 12 h/día, respectivamente (Figura $1 \mathrm{~A}$ y Tabla 3). Al ajustar estos análisis por las variables de confusión, la asociación entre horas de sueño

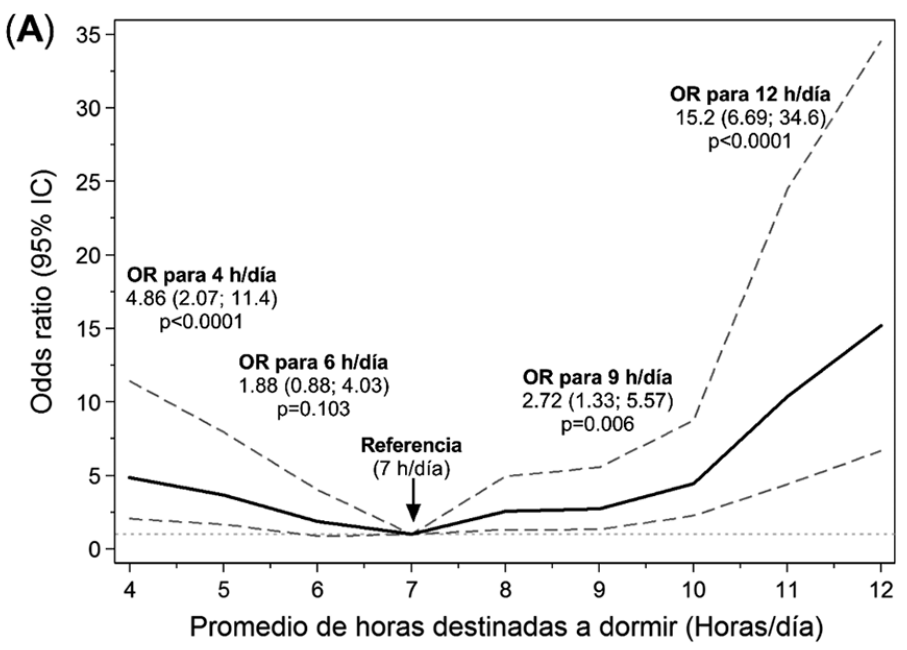

(B)

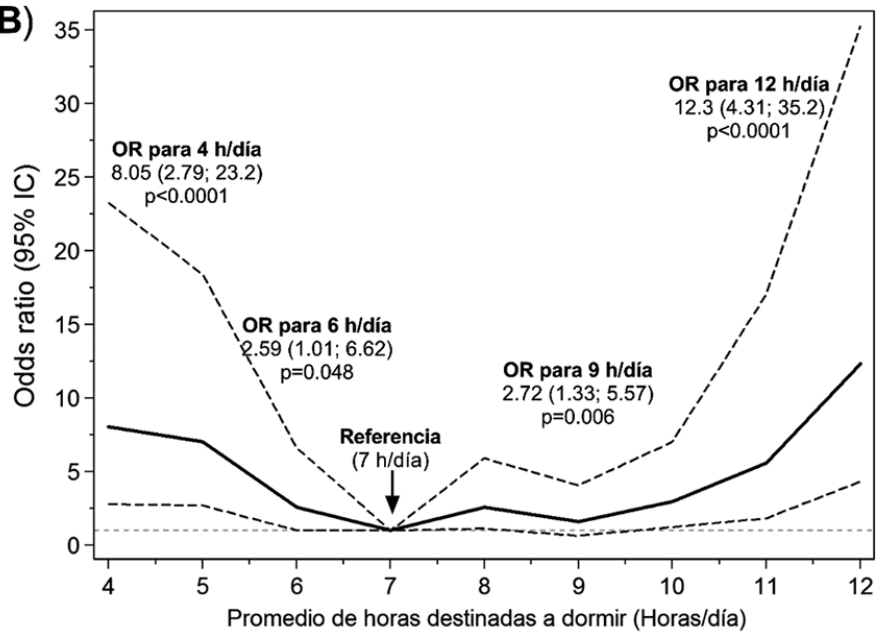

Figura 1. Asociación entre tiempo destinado a dormir y deterioro cognitivo en población adulta mayor en Chile. Datos presentados como odds ratio (OR) y sus respectivos $95 \%$ IC. El grupo de referencia fue asignado a personas que reportaron dormir en promedio $7 \mathrm{~h}$ /día. Un valor de OR mayor a 1 indica que hay un mayor riesgo de deterioro cognitivo (MMSE < 13). La figura (A) presenta resultados no ajustados, mientras que la Figura (B) presenta OR ajustados por edad, sexo, nivel educacional, tabaquismo, IMC, DMT2, HTA, AF, tiempo sedente, consumo de alcohol frutas y verduras. 
Tabla 3. Riesgo de deterioro cognitivo según horas destinadas a dormir en población adulta mayor en Chile

\begin{tabular}{|lcccc|}
\hline & \multicolumn{2}{c}{ No ajustado } & \multicolumn{2}{c|}{ Ajustado } \\
\hline Horas/día & OR (95\% IC) & Valor $\mathbf{~}$ & OR (95\% IC) & Valor p \\
\hline 4 & $4,86(2,07-11,4)$ & $<0,0001$ & $8,05(2,79-23,2)$ & $<0,0001$ \\
\hline 5 & $3,66(1,69-7,95)$ & 0,001 & $7,05(2,70-18,4)$ & $<0,0001$ \\
\hline 6 & $1,88(0,88-4,03)$ & 0,103 & $2,59(1,01-6,62)$ & 0,048 \\
\hline (Ref.) & 1,00 & - & 1,00 & 0,312 \\
\hline 8 & $2,56(1,32-4,95)$ & 0,005 & $1,61(0,64-4,06)$ & 0,025 \\
\hline 9 & $2,72(1,33-5,57)$ & 0,006 & $2,59(1,13-5,90)$ & 0,015 \\
\hline 11 & $4,46(2,27-8,74)$ & $<0,0001$ & $2,94(1,23-7,02)$ & 0,003 \\
\hline 12 & $10,3(4,40-24,4)$ & $<0,0001$ & $5,58(1,83-17,0)$ & $<0,0001$ \\
\hline
\end{tabular}

Datos presentados como odds ratio (OR) y sus respectivos $95 \%$ IC. El grupo de referencia fue asignado a personas que reportaron dormir en promedio $7 \mathrm{~h} /$ día. Un valor de OR mayor a 1 indica que hay un mayor riesgo de deterioro cognitivo (MMSE $<$ 13). OR para el modelo ajustado incluye las siguientes variables: edad, sexo, nivel educacional, tabaquismo, IMC, DMT2, $\mathrm{HTA}$, AF, tiempo sedente, consumo de alcohol, frutas y verduras.

y sospecha de deterioro cognitivo se mantuvo significativa (Figura 1B y Tabla 3). Sin embargo, las magnitudes de las asociaciones se incrementaron en quienes reportaron dormir $4 \mathrm{~h} /$ día, llegando a presentar 8 veces mayor probabilidad de tener deterioro cognitivo que personas que reportaron dormir 7 h/día (Figura 1B y Tabla 3). En el caso de los adultos mayores que reportaron dormir más de $7 \mathrm{~h}$ /día, las asociaciones fueron de menor magnitud, no obstante, se mantuvieron significativas. Personas que reportaron dormir $11 \mathrm{~h}$ /día presentaron una probabilidad 5,6 veces más alta de tener deterioro cognitivo en comparación al grupo de referencia, probabilidad que aumentó 12,3 veces para quienes informaron dormir $12 \mathrm{~h} /$ día (Figura 1B y Tabla 3).

\section{Discusión}

Este estudio confirma la asociación entre horas de sueño y deterioro cognitivo en personas adultas mayores chilenas, similar a lo descrito previamente en otras poblaciones ${ }^{12-14}$ evidenciando que tanto horas aumentadas de sueño ( $\geq 9 \mathrm{~h}$ /día) como disminuidas ( $\leq 6 \mathrm{~h} /$ día) se asocian con deterioro cognitivo en personas mayores de 60 años. Estas asociaciones fueron independientes de factores de confusión.
Los resultados de este estudio apoyan otros hallazgos que indican una asociación en forma de $U$ entre horas de sueño y funcionamiento cognitivo global $^{11,13,14,20}$. Estudios transversales, en adultos mayores españoles ${ }^{21} \mathrm{y}$ norteamericanos ${ }^{22}$, indican una asociación entre más de $9 \mathrm{~h}$ de sueño/día y disminución del funcionamiento cognitivo global $y$ en otros dominios de funcionamiento cognitivo, como la función ejecutiva y memoria. Estudios longitudinales en adultos mayores europeos reportan que horas aumentadas y disminuidas de sueño se asociaron con un menor funcionamiento cognitivo global ${ }^{23}$. Horas de sueño disminuidas estaban asociadas a un incremento de $75 \%$ de riesgo de demencia y $50 \%$ de enfermedad de Alzheimer a 9 años de seguimiento ${ }^{24}$. Por otro lado, Ramos y cols., en población americana, predominantemente de origen hispano, corroboraron la asociación entre $\geq 9 \mathrm{~h}$ de sueño/día y una menor puntuación en MMSE, pero no así con horas de sueño disminuidas ${ }^{25}$. A partir de la información disponible y la revisión realizada no se encontraron estudios en población chilena que relacionen ambas variables. Considerando que en Chile, según la ENS 2009-2010, el promedio de horas de sueño de la población mayor de 60 años se encuentra por debajo de lo recomendado, que $63,2 \%$ de las personas presenta sospecha de trastorno del sueño y que $4,6 \%$ sospecha de síndrome 
de apnea obstructiva del sueño con un incremento significativo a medida que aumenta la edad ${ }^{5}, \mathrm{los}$ resultados de este estudio podrían incentivar políticas públicas que busquen atender las implicancias de estas condiciones en la prevención de enfermedades neurocognitivas. En este contexto, estos hallazgos sugieren la necesidad de profundizar en los mecanismos neuronales que subyacen a la relación sueño y funciones mentales superiores. Adicionalmente, existe evidencia de que horas aumentadas y disminuidas de sueño se asocian a sintomatología ansiosa-depresiva, depresión y otros trastornos del ánimo ${ }^{26}$ y que a su vez estas patologías inciden en el funcionamiento cogniti$\mathrm{vo}^{27}$, por lo que es necesario estudiar el potencial efecto sinérgico entre estas condiciones.

Si bien este estudio confirma la asociación entre horas de sueño y deterioro cognitivo, es importante considerar ciertas limitaciones metodológicas al momento de interpretar estos hallazgos. Entre las limitaciones está el uso de cuestionario para la medición de horas destinadas a dormir, con los potenciales errores de medición asociados al auto-reporte. Estudios futuros deberían incluir la medición de horas de sueño mediante técnicas objetivas como acelerómetros de movimiento. Otra limitante es la falta de información sobre la calidad de sueño, trastornos del sueño y registro de consumo de medicación, lo cual podría ser un importante factor de confusión en los resultados. Por otra parte, el MMSE ha sido objeto de evaluación respecto a su sensibilidad en la detección de deterioro ${ }^{28}$ por lo que el presente estudio solo puede hacer referencia a funcionamiento cognitivo alterado y sospecha de deterioro. También es importante considerar que la población estudiada se estratificó por sospecha de deterioro cognitivo, por lo cual la precisión en el auto-reporte de las variables estudiadas (ej. Estilos de vida) pudo haber sido menor en personas con deterioro cognitivo. Entre las fortalezas del estudio destacan que es el primero realizado en población chilena que responde a esta pregunta de investigación. Si bien los métodos utilizados fueron en su mayoría auto reportados la implementación de protocolos estandarizados y representatividad nacional permiten extrapolar estos resultados a la población adulta mayor en Chile.

Este estudio confirma la asociación previamente descrita entre horas de sueño diarias y deterioro cognitivo en población chilena. Dado el aumento de la esperanza de vida se prevé un aumento sostenido del deterioro cognitivo y demencias por lo que estas patologías aparecen dentro de los principales desafíos de la salud pública mundial. Las horas de sueño juegan un rol importante en el funcionamiento cerebral a medida que se envejece y conforman un factor de riesgo modificable posible de intervenir para evitar deterioro cognitivo y su evolución hacia demencia. Promover adecuados hábitos de sueño debería ser considerado en los programas preventivos dirigidos a la población adulta mayor chilena.

Agradecimientos: Se agradece de manera especial a todos los participantes de la ENS 20092010, al equipo profesional de la Escuela de Salud Pública, de la Facultad de Medicina de la Pontificia Universidad Católica de Chile, quienes desarrollaron y aplicaron la Encuesta Nacional de Salud y al Ministerio de Salud del Gobierno de Chile.

\section{Referencias}

1. World Health Organization. Dementia. A Public Health Priority. 2012. Disponible en: http://apps.who.int/iris/ bitstream $/$ handle/10665/75263/978? sequence $=1$ [Consultado el 5 de agosto de 2018].

2. Sachdev PS, Lipnicki DM, Kochan NA, Crawford JD, Thalamuthu A, Andrews G, et al. The Prevalence of Mild Cognitive Impairment in Diverse Geographical and Ethnocultural Regions: The COSMIC Collaboration. PLoS One 2015 11; 10 (11).

3. Maioli F, Coveri M, Pagni P, Chiandetti C, Marchetti C, Ciarrocchi R, Ruggero C, et al. Conversion of mild cognitive impairment to dementia in elderly subjects: a preliminary study in a memory and cognitive disorder unit. Arch Gerontol Geriatr 2007; 44: 233-41.

4. Xu J, Zhang Y, Qiu C, Cheng F. Global and regional economic costs of dementia: a systematic review. The Lancet 2017; 390: S47.

5. MINSAL. Chile: Encuesta Nacional de Salud 20092010. Ministerio de Salud de Chile. 2010; Disponible en: http://www.minsal.cl/portal/url/item/bcb03d7bc28b64dfe040010165012d23.pdf[Consultadohttp:// web.minsal.cl/portal/url/item/bcb03d7bc28b64dfe040010165012d23.pdf [Consultado el 20 de agosto de 2018].

6. MINSAL. Plan Nacional de Demencia 2017. Ministerio de Salud de Chile. Disponible en: http://www.minsal.cl/ 
wp-content/uploads/2017/11/PLAN-DE-DEMENCIA. pdf [Consultado el 15 de agosto de 2018].

7. Cooper C, Sommerlad A, Lyketsos CG, Livingston G. Modifiable predictors of dementia in mild cognitive impairment: a systematic review and metaanalysis. Am J Psychiatry 2015; 172 (4): 323-34.

8. Li JQ, Tan L, Wang HF, Tan MS, Tan L, Xu W, et al. Risk factors for predicting progression from mild cognitive impairment to Alzheimer's disease: a systematic review and meta-analysis of cohort studies. J Neurol Neurosurg Psychiatry 2016; 87 (5): 476-84.

9. Livingston $\mathrm{G}$, Sommerlad A, Orgeta V, Costafreda SG, Huntley J, Ames D, et al. Dementia prevention, intervention, and care. The Lancet 2017; 390 (10113): 2673734. Disponible en: http://www.ucl.ac.uk/psychiatry/ research/olderpeople/lancet-dementia-commission [Consultado el 5 de agosto de 2018].

10. Yaffe K, Falvey CM, Hoang T. Connections between sleep and cognition in older adults. Lancet Neurol 2014; 13 (10): 1017-28.

11. Devore EE, Grodstein F, Duffy JF, Stampfer MJ, Czeisler CA, Schernhammer ES. Sleep duration in midlife and later life in relation to cognition. J Am Geriatr Soc 2014; 62 (6): 1073-81.

12. Kronholm E, Sallinen M, Suutama T, Sulkava R, Era P, Partonen T. Self-reported sleep duration and cognitive functioning in the general population. J Sleep Res 2009; 18 (4): 436; 46.

13. Virta JJ, Heikkilä K, Perola M, Koskenvuo M, Räihä I, Rinne JO, et al. Midlife sleep characteristics associated with late life cognitive function. Sleep 2013; 1; 36 (10): 1533-41.

14. Benito León J, Louis ED, Bermejo-Pareja F. Cognitive decline in short and long sleepers: a prospective population; based study (NEDICES). J Psychiatr Res. 2013; 1; 47 (12): 1998-2003.

15. WHO. Informe Mundial sobre el envejecimiento y la salud. World Health Organization, 2015. Disponible en: http://www.who.int/ageing/publications/world-report-2015/es/ [Consultado el 2 de agosto de 2018].

16. Folstein MF, Folstein SE, McHugh PR. Mini-Mental State: a practical method for grading the cognitive state of patients for the clinician. J Psychiatr Res 1975; 12: 189-98.

17. Icaza MG, Abdala C. Minimental State Examination: Análisis estadístico del estudio de demencia en Chile para validar una versión abreviada. Investigaciones en Salud Pública: Documento Técnico. OPS, Washington, DC. 1999. Disponible: http://www.bvsde.paho.org/ texcom/ [Consultado el 20 de agosto de 2018].

18. Quiroga P, Albala C, Klaasen G. Validación de un test de tamizaje para el diagnóstico de demencia asociada a edad en Chile. Rev Med Chile 2004; 132: 467-78.

19. WHO. Global Physical Activity Questionnaire: GPAQ version 2.0. World Health Organization. 2009. Disponible en: http://www.who.int/chp/steps/resources/ GPAQ_Analysis_Guide.pdf. [Consultado el 2 de agosto de 2018].

20. Lo JC, Groeger JA, Cheng GH, Dijk DJ, Chee MW. Self- reported sleep duration and cognitive performance in older adults: a systematic review and meta-analysis. Sleep med 2016; 17: 87-98.

21. Faubel R, López-García E, Guallar-Castillón P, Graciani A, Banegas JR, Rodríguez-Artalejo F. Usual sleep duration and cognitive function in older adults in Spain. J Sleep Res 2009; 18 (4): 427-35.

22. Blackwell T, Yaffe K, Ancoli-Israel S, Redline S, Ensrud KE, Stefanick ML, et al. Association of sleep characteristics and cognition in older community; dwelling men: the MrOS Sleep Study. Sleep 2011; 1; 34 (10): 1347-56.

23. Van Oostrom SH, Nooyens AC, van Boxtel MP, Verschuren WM. Long sleep duration is associated with lower cognitive function among middle-age adults, the Doetinchem Cohort Study. Sleep Med 2018; 41: 78-85.

24. Hahn EA, Wang HX, Andel R, Fratiglioni L. A change in sleep pattern may predict Alzheimer disease. Am. J. Geriatr. Psychiatry 2014; 22: 1262e127.

25. Ramos AR, Dong C, Elkind MSV, Boden-Albala B, Sacco RL, Rundek T, et al. Association between Sleep Duration and the Mini-Mental Score: The Northern Manhattan Study. J Clin Sleep Med 2013; 9 (7): 669-73.

26. Alvaro PK, Roberts RM, Harris JK. A systematic review assessing bidirectionality between sleep disturbances, anxiety, and depression. Sleep 2013; 36 (7): 1059-68.

27. Rock PL, Roiser JP, Riedel WJ, Blackwell AD. Cognitive impairment in depression: a systematic review and meta-analysis. Psychol Med 2014; 44 (10): 2029-40.

28. Jiménez D, Lavados M, Rojas P, Henríquez C, Silva F, Guillon M. [Performance of an abbreviated mini mental examination to detect dementia in older people]. Rev Med Chile 2017; 145 (7): 862-8. 